GANIT J. Bangladesh Math. Soc. (ISSN 1606-3694) 39 (2019) 119-126

DOI: https://doi.org/10.3329/ganit.v39i0.44163

\title{
SYMPLECTIC AND CONTACT GEOMETRY WITH COMPLEX MANIFOLDS
}

\author{
A. K. M. Nazimuddin ${ }^{1, *}$ and Md. Showkat $\mathrm{Ali}^{2}$ \\ ${ }^{1}$ Department of Mathematical and Physical Sciences, East West University, Dhaka-1212 \\ ${ }^{2}$ Department of Applied Mathematics, University of Dhaka, Dhaka-1000 \\ *Corresponding author:nazimuddin@ewubd.edu
}

Received: 28-04-2018

Accepted: 11-11-2019

\begin{abstract}
In this paper, we discuss about almost complex structures and complex structures on Riemannian manifolds, symplectic manifolds and contact manifolds. We have also shown a special comparison between complex symplectic geometry and complex contact geometry. Also, the existence of a complex submanifold of $\mathrm{n}$-dimensional complex manifold which intersects a real submanifold.
\end{abstract}

Keywords: Symplectic, Contact, Complex manifold.

\section{Introduction}

Thurston[18] gave the example of compact symplectic manifold where Kähler structure doesn't exist. Thurston's example had already been discovered as a complex manifold, by kodaira during his work on the classification of compact complex surfaces [4]. complex contact manifold was discovered based on the works of Kobayashi and Boothby [5], [6], [7]. Then, homogeneous complex contact manifoldsare studied in [19]. Normality for complex contact structures are discussed in [2], [3] and a new result for the normality is given by [8], [9], [10]. Recently, Nazimuddin and Ali developed various connections with symplectic structures [14].

\section{Complex Symplectic Manifolds}

Let $(M, \omega)$ be a complex-symplectic manifold with $\operatorname{dim}_{\mathbb{C}} M=2 n$ and complex structure $J_{0}$. Then $\omega$ is a closed, holomorphic 2-form with $\omega^{n} \neq 0$. Let $\omega=\omega_{1}+i \omega_{2}$, where $\omega_{1}$ and $\omega_{2}$ are real 2forms. Since $\omega$ is closed, so are $\omega_{1}$ and $\omega_{2}$. Also, $\omega$ being holomorphic means that $\omega\left(X+i J_{0} X\right.$,* ) $=0$ as a 1 -form on $T^{\mathbb{C}} M$.It is easy to see then that

$$
\omega_{2}(X, Y)=-\omega_{1}\left(J_{0} X, Y\right)=-\omega_{1}\left(X, J_{0} Y\right)
$$

for any real vectors $\mathrm{X}$ and $\mathrm{Y}$. Now, we may use the complex version of Darboux's Theorem to find local holomorphic functions $\left(z_{1}, \ldots, z_{n}, w_{1}, \ldots, w_{n}\right)$ such that $\omega=d z_{1} \wedge d w_{1}+\cdots+d z_{n} \wedge$ $d w_{n}$. If we derive real coordinates $z_{j}=x_{j}+i y_{j}, w_{j}=s_{j}+i t_{j}$, then 


$$
\begin{aligned}
& \omega_{1}=d x_{1} \wedge d s_{1}-d y_{1} \wedge d t_{1}+\cdots+d x_{n} \wedge d s_{n}-d y_{n} \wedge d t_{n} \\
& \omega_{2}=d x_{1} \wedge d t_{1}-d y_{1} \wedge d s_{1}+\cdots+d x_{n} \wedge d t_{n}-d y_{n} \wedge d s_{n}
\end{aligned}
$$

from which we see that $\omega_{1}^{2 n} \neq 0$ and $\omega_{2}^{2 n} \neq 0$. Thus, we have two distinct symplectic structures on $\mathrm{M}$.

Gromovdemonstrated that always carries a compatible symplectic structure [1] contains an open almost complex manifold M. For compact manifolds, existence of an almost complex structure does not imply existence of a symplectic structure and the simplest additional necessary conditionis the existence of a closed 2-form $\omega$ such that its powers $\omega^{\mathrm{j}}$ are cohomologically for $=1, \cdots, N:[\omega]^{j} \neq 0$ in $H^{2 j}(M)$. A complex manifold $\mathrm{M}$ is called a Kähler manifold if it carries a Hermitian metric $\mathrm{h}_{\mathrm{ij}} \mathrm{dz} \mathrm{z}^{\mathrm{i}} \mathrm{d} \overline{\mathrm{z}}^{\mathrm{j}}$ such that the form $\omega=h_{i j} d z^{i} d \bar{z}^{j}$ is closed. This form is symplectic and therefore any Kähler manifold carries a natural symplectic structure.

The simplest examples of Kähler manifolds are algebraic manifolds which are complex submanifolds of the complex projective spaces. For such manifold a Kähler structure is given by the metric induced from the Fubiny-Study metric by the embedding. Denote by $\left(\mathbb{C P}^{n}, \omega_{F S}\right)$ the complex projective space $\mathbb{C P}^{\mathrm{n}}$ with a Kähler form $\omega_{\mathrm{FS}}$ induced by the Fubiny-Study metric. These symplectic manifolds serve as universal symplectic manifolds in the following sense.

Proposition 2.1. [17]Let $(M, \omega)$ be a compact symplectic manifold of dimension $2 \mathrm{n}$ such that the form $\omega$ is integer, i.e. $[\omega] \in H^{2}(M ; \mathbb{Z}) \subset H^{2}(M ; \mathbb{R})$. Then there exists an embedding

$f: M \rightarrow \mathbb{C P}^{2 n+1}$ such that $f * \omega_{F S}=\omega$.

2.1 Complex symplectic structure on $T^{*} \mathcal{T}(S)$

It is important that if $M$ is any complex manifold (when $M=\mathcal{T}(S)$ ), the total space of holomorphic cotangent bundle $T^{*} M$ is equipped with a canonical complex symplectic structure.

The canonical 1 -form $\xi$ is the holomorphic $(1,0)$-form on $\mathrm{T}^{*} \mathrm{M}$ defined at a point $\phi \in T^{*} M$ by $\xi_{\phi}:=\pi^{*} \phi$, where $\pi: T^{*} M \rightarrow M$ is the canonical projection and $\phi$ is seen as a complex covector on $\mathrm{M}$ in the right-hand side of the equality. The canonical complex symplectic form on $\mathrm{T}^{*} \mathrm{M}$ is then simply defined by $\omega_{\text {can }}=d \xi$.If $\left(z_{k}\right)$ is a system of holomorphic coordinates on $\mathrm{M}$ so that an arbitrary (1,0)-form has an expression of the form $\alpha=\sum w_{k} d z_{k}$, then $\left(z_{k}, w_{k}\right)$ is a system of holomorphic coordinates on $T^{*} M$ for which $\xi=\sum w_{k} d z_{k}$ and $\omega_{\text {can }}=\sum d w_{k} \wedge d z_{k}$. The canonical 1 -form satisfies the following reproducing property. If $\alpha$ is any $(1,0)$-form on $M$, it is a map $M \rightarrow T^{*} M$ and as such it can be used to pull back differential forms from $T^{*} M$ to $M$. easy to show that $\alpha^{*} \xi=\alpha$ and consequently $\alpha^{*} \omega_{\text {can }}=d \alpha$.

\section{Complex Contact Manifolds}

Let $X$ be a complex manifold and $T X$ its holomorphic tangent bundle. The complex manifold $X$ is called contact if there is a complex-codimension one holomorphic sub-bundle $D$ of $T X$ which is 
maximally non-integrable, i.e. the tensor

$$
\begin{gathered}
D \times D \rightarrow T X / D \\
(v, w) \mapsto[v, w] \bmod D
\end{gathered}
$$

is non-degenerate for every point of $X$.

Let $L:=T X / D$ be the quotient line bundle and $\theta: T X \rightarrow L$ the tautological projection, so that we have the short exact sequence

$$
0 \rightarrow D \rightarrow T X \rightarrow L \rightarrow 0 .
$$

The projection $\theta$ is a 1-form with the line bundle $L, \theta \in \Gamma\left(X, \Omega^{1}(L)\right)$, with $\operatorname{ker}(\theta)=D$. The subbundle $D$ must have even rank $2 \mathrm{n}$ and, therefore, the manifold $X$ has odd complex dimension $2 n+$ $1 \geq 3$. Moreover, the non-degeneracy condition implies

$$
\theta \wedge(d \theta)^{n} \in \Gamma\left(X, \Omega^{2 n+1}\left(L^{n+1}\right)\right)
$$

is nowhere zero. This provides an isomorphism of the anti-canonical line bundle [11], [13] of $X$ and $L^{n+1}$. Since $L=T X / D$, there is a $C^{\infty}$ isomorphismTX $\cong D \oplus L$, so that

$$
c(X)=c(D) \cdot c(L) .
$$

There is also the following isomorphism

$$
D \cong D^{*} \otimes L
$$

Now, we can write the Chern classes

$$
c(D)=\left(1+y_{1}\right)\left(1+y_{2}\right) \cdots\left(1+y_{2 n}\right),
$$

and

$$
c(L)=\left(1+y_{2 n+1}\right),
$$

so that

$$
c_{1}(X)=(n+1) y_{2 n+1} .
$$

\subsection{General facts about global complex contact structures}

Throughout this section, we assume that $\eta=u-i v$ is a global holomorphic contact form $(u$ and $v$ are real 1-forms with $v=u \circ J$ ) and that $V$ is the subbundle of TP defined as the span of $\{U, V=-J U\}$ where

$$
\begin{aligned}
& u(U)=1, v(U)=0, \iota(U) d u=0, \\
& u(V)=0, v(V)=1, \iota(V) d v=0 .
\end{aligned}
$$

Theorem 3.1. If $P$ is a complex contact manifold with a global holomorphic contact form $\eta=u-i v$ and corresponding vertical subbundle $V=\operatorname{span}\{U, V=-J U\}$ given by 


$$
\begin{aligned}
& u(U)=1, v(U)=0, \iota(U) d u=0, \\
& u(V)=0, v(V)=1, \iota(V) d v=0 .
\end{aligned}
$$

Then

1. $U$ and $J U$ are infinitesimal automorphisms of $J$, i.e., $\mathcal{L}_{U} J=\mathcal{L}_{J U} J=0$.

2. $[U, J U]=0$, so that $V$ is a foliation of $T P$.

3. $\mathcal{L}_{U} u=\mathcal{L}_{J U} u=\mathcal{L}_{U} v=\mathcal{L}_{J U} v=0$.

4. $\mathcal{L}_{U}(d u)=\mathcal{L}_{J U}(d u)=\mathcal{L}_{U}(d v)=\mathcal{L}_{J U}(d v)=0$.

Proof. If we use the complex Darboux Theorem to derive holomorphic coordinates $\left(z_{1}, \ldots, z_{2 n+1}\right)$ such that $\eta=d z_{1}-z_{2} d z_{3}-\cdots-z_{2 n} d z_{2 n+1}$, then we see immediately that $\frac{1}{2}(U-i J U)=$ $\partial / \partial z_{1}$. In other words, both $U=\partial / \partial x_{1}$ and $J U=\partial / \partial y_{1}$ are infinitesimal automorphisms of $J$ So, $\mathcal{L}_{U} J=\mathcal{L}_{J U} J=0$. In particular, $[U, J U]=J[U, U]=0$, i.e., $V$ is a foliation. Also, note that, on each vertical leaf, we have a hermitian metric given by

$$
g^{\prime}=u \otimes u+v \otimes v,
$$

i.e., $U$ and $J U$ are taken to be orthonormal vector fields. By assumption, $d \eta$ is a holomorphic 2form on $P$. In particular,

$$
d v(X, Y)=d u(J X, Y)=d u(X, J Y)
$$

for any vectors $X, Y$ on $P$.we also have $d v(U, X)=d u(U, J X)=0$ and similarly, $d u(V, X)=0$ for any $X \in T P$. Thus, if $X \in H$, then

$$
v([U, X])=-2 d v(U, X)=0, u([U, X])=-2 d u(U, X)=0 .
$$

So, $[U, X] \in \mathcal{H}$. Similarly, $[V, X] \in \mathcal{H}$. Furthermore, for any $z \in P$, there is an open subset $Y$ of $P$ such that the space $\frac{Y}{V}$, the space of maximal vertical leaves on $Y$ given the quotient topology, is an open manifold and $P_{Y}: Y \rightarrow \frac{Y}{V}$ is a submersion. Then, for any basic vector field $X$ on $Y$, i.e., $X$ is horizontal and $\left(P_{Y}\right)_{*} X$ is a well-defined vector field on $\frac{Y}{V}$, we have

$$
\left(P_{Y}\right)_{*}([U, X])=\left(P_{Y}\right)_{*}([V, X])=0 .
$$

So, $[U, X]$ and $[V, X]$ are also vertical. Thus, $[U, X]=[V, X]=0$.

If $X$ is any horizontal vector and we extend $X$ to be a local basic vector field on $P$, then

$$
\mathcal{L}_{U} u(X)=-u([U, X])=0 .
$$

Hence, $\mathcal{L}_{U} u=0$. Similarly, we have $\mathcal{L}_{U} v=0=\mathcal{L}_{J U} v=\mathcal{L}_{J U} u$.

Using this same argument, we have

$$
\mathcal{L}_{U}(d u)=\mathcal{L}_{J U}(d u)=\mathcal{L}_{U}(d v)=\mathcal{L}_{J U}(d v)=0 .
$$

This completes the proof. 


\section{Comparison between Complex Symplectic Geometry and Complex Contact Geometry}

\begin{tabular}{|c|c|}
\hline Complex Symplectic Geometry & Complex Contact Geometry \\
\hline $\begin{array}{l}\text { 1. Complex Symplectic Manifold } \\
\text { The complex manifold } X \text { of complex dimension } 2 n \\
\text { is called symplectic if it has a holomorphic } \\
\text { symplectic } 2 \text {-form } \omega \text { is closed with } \omega^{n} \neq 0 \text {. } \\
\text { Let } \omega=\omega_{1}+i \omega_{2} \\
\text { These two closed forms } \omega_{1} \text { and } \omega_{2} \text { are real } \\
\text { symplectic forms and define the structure of a } \\
\text { complex symplectic manifold on } X \text {. }\end{array}$ & $\begin{array}{l}\text { 1. Complex Contact Manifold } \\
\text { The complex manifold } X \text { of complex dimension } \\
2 n+1 \text { is called contact if there is a complex co- } \\
\text { dimension one holomorphic sub-bundle Dof } \\
T X \text { which is maximally non-integrable, i.e. the } \\
\text { tensor } \\
\qquad D \times D \rightarrow T X / D \\
(v, w) \mapsto[v, w] \bmod D \\
\text { is non-degenerate for every point of } X .\end{array}$ \\
\hline $\begin{array}{l}\text { 2. Examples } \\
\text { (i) Kodaira- Thurston manifold represents a } \\
\text { complex symplectic manifold. Let } \mathrm{g} \text { be the Lie } \\
\text { algebra of } G \text { and let } \mathrm{g}^{*} \text { be its dual. We identify } \\
\text { tensors on } \mathrm{g} \text { and } \mathrm{g}^{*} \text { with left-invariant objects on } G \text {. } \\
\text { It is easy to check that } \mathrm{g} \text { has a basis }<X_{1}, X_{2}, X_{3} \text {, } \\
X_{4}>\text { in which the only non-zero bracket } \\
\text { is }\left[X_{1}, X_{2}\right]=-X_{3} \text {. Let }<x_{1}, x_{2}, x_{3}, x_{4}>\text { be the } \\
\text { dual basis of } \mathrm{g}^{*} \text {. The only non-zero differential on } \\
\mathrm{g}^{*} \text { is computed to be } d x_{3}=x_{1} \wedge x_{2} \text {. The element } \\
\omega=x_{1} \wedge x_{4}+x_{2} \wedge x_{3} \text { is closed and non-degenerate. }\end{array}$ & $\begin{array}{l}\text { 2. Examples } \\
\text { (i) The odd-dimensional complex projective space } \\
\mathbb{P}^{2 n+1} \text { is a complex contact manifold. Any 2- } \\
\text { homogeneous symplectic form } \omega \text { on } \mathbb{C}^{2 n+2} \text { defines } \\
\text { a contact form on } \mathbb{P}^{2 n+1} \text {. }\end{array}$ \\
\hline $\begin{array}{l}\text { (ii) Consider the holomorphic Lie groups } \\
\mathbb{C}^{3} \cong H_{\mathbb{C}}=\left\{\left(\begin{array}{ccc}1 & z_{2} & z_{1} \\
0 & 1 & z_{3} \\
0 & 0 & 1\end{array}\right): z_{1}, z_{2}, z_{3} \in M_{3 \times 3}(\mathbb{C})\right\} \\
\text { and } \mathbb{C}^{2}=\left(\begin{array}{l}z_{1} \\
z_{1}\end{array}\right) \text {. Then the map } \pi: \mathbb{C}^{3} \rightarrow \mathbb{C}^{2} \text { is a } \\
\text { surjective holomorphic Lie group homomorphism. } \\
\text { The holomorphic 2-form } \omega=-d z_{1} \wedge d z_{2} \text { on } \mathbb{C}^{2} \text { is } \\
\text { a left-invariant complex symplectic form. }\end{array}$ & $\begin{array}{l}\text { (ii)Complex Heisenberg group } H_{\mathbb{C}} \text { represents a } \\
\text { complex contact manifold, where } \\
\qquad\left\{\left(\begin{array}{ccc}1 & z_{2} & z_{1} \\
0 & 1 & z_{3} \\
0 & 0 & 1\end{array}\right): H_{\mathbb{C}}=\right. \\
\text { The complex contact structure of this manifold is } \\
\text { given by the left invariant } 1 \text {-form } \\
\eta=d z_{1}-z_{2} d z_{3} \text { and } \eta \wedge d \eta \neq 0\end{array}$ \\
\hline 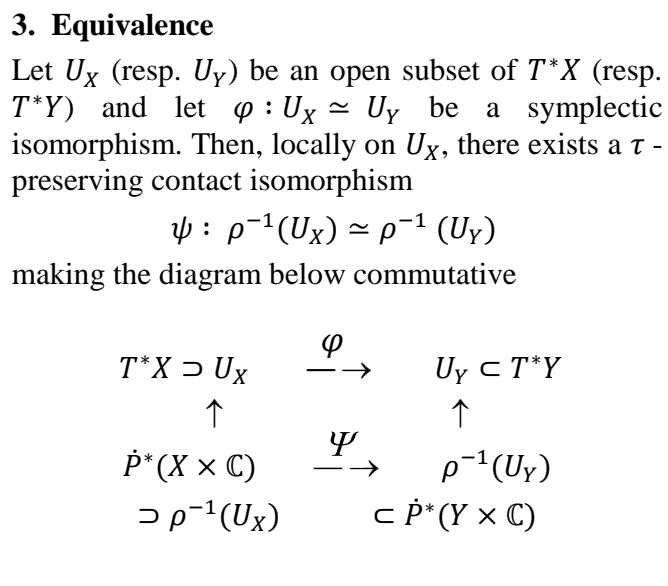 & 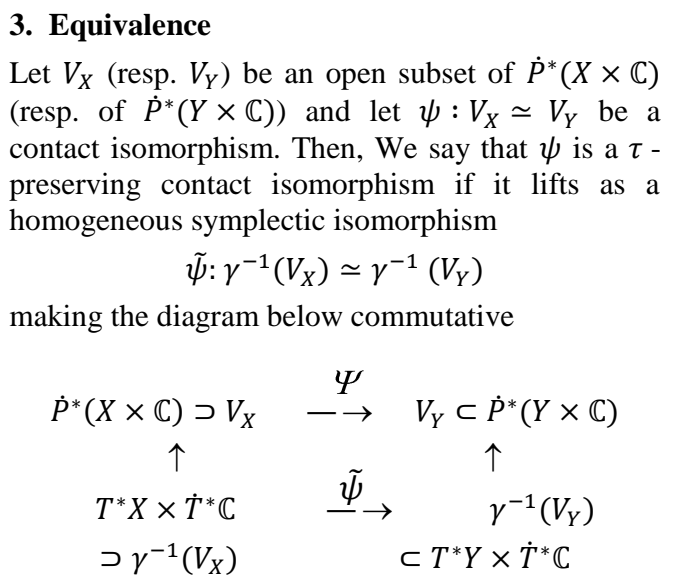 \\
\hline
\end{tabular}




\begin{tabular}{|c|c|}
\hline $\begin{array}{l}\text { 4. Quantization-deformation modules } \\
\text { Let } X \text { be a complex symplectic manifold. There } \\
\text { exists canonically a } K \text {-Abelian stack } \bmod \left(\mathcal{W}^{\sqrt{v}}, X\right) \\
\text { on } X \text { such that if } U \subset X \text { is an open subset } \\
\text { isomorphic by a contact transformation } \varphi \text { to an } \\
\text { open subset } U_{X} \subset T^{*} X \text {, then }\left.\bmod \left(\mathcal{W}^{\sqrt{v}}, X\right)\right|_{U} \text { is } \\
\text { equivalent by } \varphi \text { to the stack } \bmod \left(\mathcal{W}_{X}^{\sqrt{v}} \mid U_{X}\right) \text {. }\end{array}$ & $\begin{array}{l}\text { 4. Quantization-deformation modules } \\
\text { Let } Y \text { be a complex contact manifold. There exists } \\
\text { canonically a } \mathbb{C} \text {-Abelian stack } \bmod \left(\varepsilon^{\sqrt{v}}, Y\right) \text { on } Y \\
\text { such that if } V \subset Y \text { is an open subset isomorphic by } \\
\text { a contact transformation } \psi \text { to an open subset } \\
V_{Y} \subset P^{*} Y \text {, then }\left.\bmod \left(\varepsilon^{\sqrt{v}}, Y\right)\right|_{V} \text { is equivalent by } \psi \\
\text { to the stack } \bmod \left(\varepsilon_{Y}^{\sqrt{v}} \mid V_{Y}\right) \text {. }\end{array}$ \\
\hline 5. Local Chara & 5. Local Character \\
\hline $\begin{array}{l}\text { Let } X \text { be a complex symplectic manifold. Now } \\
\text { Darboux's theorem implies that, the local model of } \\
X \text { is an open subset of the cotangent bundle } \\
T^{*} M \text { with } M=\mathbb{C}^{\frac{1}{2} \operatorname{dim} X} \text {. }\end{array}$ & $\begin{array}{l}\text { Let } Y \text { be a complex contact manifold. Now } \\
\text { Darboux's theorem implies that, the local model of } \\
Y \text { is an open subsetof the projective cotangent } \\
\text { bundle } P^{*} M \text { with } M=\mathbb{C}^{\frac{1}{2}(\operatorname{dim} Y+1)} \text {. }\end{array}$ \\
\hline
\end{tabular}

\section{Existence of complex submanifolds}

We are interested in complex submanifolds $S$ in $\mathbb{C}^{2 n}$ that intersect the real submanifold $M$ at the origin. Generically, the origin is an isolated intersection point if $\operatorname{dim} S=n$. Let us consider the situation when the intersection has dimension $n$. Without further restrictions, there are many such complex submanifolds; for instance, we can take a $n$-dimensional totally real and real analytic submanifold $S_{1}$ of $M$. We then let $S$ be the complexification of $S_{1}$. To ensure the uniqueness or finiteness of the complex submanifolds $S$, we therefore introduce the following.

Definition 4.1.Let Mbe a formal real submanifold of dimension $2 \mathrm{nin} \mathbb{C}^{2 \mathrm{n}}$. We say that a formal complex submanifold $S$ is attached to $M$ if $S \cap M$ contains at least two germs of totally real and formal submanifolds $S_{1}, S_{2}$ of dimension $n$ that intersect transversally at the origin and $S$ has dimension $n$. Such a pair $\left\{S_{1}, S_{2}\right\}$ are called a pair of asymptotic formal submanifolds of $M$.

We first derive the results at the formal level. We then apply the results of [15], [16]. The proof of the co-existence of convergent and divergent attached submanifolds will rely on a theorem of Pöschel on stable invariant submanifolds and Siegel's small divisor technique. We now describe the formal results. When $n=1$, a non-resonant hyperbolic Madmits a unique attached formal holomorphic curve [12]. When $n>1$, new situations arise. First, we show that there are obstructions to attach formal submanifolds. However, the formal obstructions disappear when Madmits the maximum number of deck transformations and $M$ is non-resonant. We will consider a real submanifold $M$ which is a higher order perturbation of a non-resonant product quadrics. By adapting the proof of Klingenberg to the manifold $M$, we will show the existence of a unique attached formal submanifold for a prescribed non-resonance condition. We also show that the complexification of $S$ in $M^{\prime}$ is a pair of invariant formal submanifolds $S^{\prime}{ }_{1}, S^{\prime}{ }_{2}$ of $\sigma$. Furthermore, Sis convergent if and only if $S^{\prime}{ }_{1}$ is convergent. We now can prove the following theorem.

Theorem 5.1. Let $M$ be a real analytic submanifold in $\mathbb{C}^{2 n}$ without elliptic components. Assume that in $(\xi, \eta)$ coordinates, $D \sigma(0)$ is diagonal and has distinct eigenvalues $\mu_{1}, \mu_{2}, \ldots, \mu_{n}, \mu_{1}^{-1}, \mu_{2}{ }^{-1}, \ldots, \mu_{n}{ }^{-1}$. Let $v=v_{\varepsilon}$, Then $M$ admits a unique pair of formal asymptotic 
submanifold $\left\{S^{\varepsilon}{ }_{1}, S^{\varepsilon}{ }_{2}\right\}$ such that the complexification of $S^{\varepsilon}{ }_{1}$ in $M^{\prime}$ is an invariant formal submanifold $H_{\varepsilon}$ of $\sigma$ that is tangent to

$$
\cap_{\varepsilon_{j}=1}\left\{\eta_{j}=0\right\} \cap \cap_{\varepsilon_{j}=-1}\left\{\xi_{j}=0\right\} .
$$

Furthermore, the complexification of $S^{\varepsilon}{ }_{2}$ equals $\tau_{1} H_{\varepsilon}$.

Proof. Let $S_{i}=S^{\varepsilon}{ }_{i}$. We will follow Klingenberg's approach for $n=1$, by using the deck transformations. Suppose that $S$ is an attached formal complex submanifold which intersects with $M$ at two totally real formal submanifolds $S_{1}, S_{2}$. We first embed $S_{1} \cup S_{2}$ into $M^{\prime}$ as $M$ is embedded into $M^{\prime}$. Let $S^{\prime}{ }_{i}$ be the complexification of $S_{i}$ in $M^{\prime}$. Since $\rho$ fixes $S_{i}$ pointwise, then $\rho S_{i}=S_{i}$.

We want to show that $\tau_{1}\left(S^{\prime}{ }_{1}\right)=S^{\prime}{ }_{2}$; thus $S^{\prime}{ }_{i}$ is invariant under $\sigma$. We can see that $S^{\prime}{ }_{i}$ is defined by

$$
\bar{\rho}_{l}\left(z^{\prime}\right)=w^{\prime}
$$

On $S^{\prime}{ }_{1}$, we have $L\left(z^{\prime}, w^{\prime}\right)+E\left(z^{\prime}, w^{\prime}\right)=-f\left(z^{\prime}\right)$. The latter defines a complex submanifold of dimension $n$. Thus it must be $S^{\prime}{ }_{1}$. On $M^{\prime}$,

$$
\left(L_{j}\left(z^{\prime}, w^{\prime}\right)+E_{j}\left(z^{\prime}, w^{\prime}\right)\right)^{2}=z_{p+j}
$$

are invariant by $\tau_{1}$. Thus each $L_{j}\left(z^{\prime}, w^{\prime}\right)+E_{j}\left(z^{\prime}, w^{\prime}\right)$ is either invariant or skew-invariant by $\tau_{1}$. Computing the linear part, we conclude that they are all skew-invariant by $\tau_{1}$. Hence $\tau_{1}\left(S^{\prime}{ }_{1}\right)$ is defined by $L_{j}\left(z^{\prime}, w^{\prime}\right)+E_{j}\left(z^{\prime}, w^{\prime}\right)=f\left(z^{\prime}\right)$, which is the defining equations for $S^{\prime}{ }_{2}$. We must identify the tangent space of $S^{\prime}{ }_{1}$ at the origin. Finally, if $S^{\prime}{ }_{1}$ is convergent, then $\overline{\rho_{1}}$ is convergent. Hence $S^{\prime}{ }_{1}$, the fixed point set of $\rho_{1}$, is convergent.

\section{Conclusion}

In this study, symplectic and contact geometry with complex manifolds are investigated. We have discussed about canonical complex symplectic structures and the general facts about complex contact structures. The main point in this study is the special comparison of complex symplectic geometry and complex contact geometry.

\section{REFERENCES}

[1] Gromov, M. L., A topological technique for the construction of solutions of diffdrerential equations and inequalities, Actes Congres Intern. Math., Gauthier-Villars, Paris, 2 (1971), 221-225.

[2] Ishihara S. and Konishi M., Real contact 3-structure and complex contact structure, Southeast Asian Bulletin of Math, 3 (1979), 151-161.

[3] Ishihara S. and Konishi M., Complex almost contact manifolds, Kodai Math. J., 3 (1980), 385-396.

[4] Kodaira K., On the structure of compact complex analytic surfaces. I, Amer. J. Math. 86 (1964), 751798.

[5] Kobayashi, S., Principal fibre bundles with the1-dimensional toroidal group, Tohoku Math. J. 8 (1956), 29-45.

[6] Kobayashi S., Remarks on complex contact manifolds, Proc. Amer. Math. Soc., 10 (1963), 164-167. 
[7] Kobayashi S., Topology of positively pinched Kähler manifolds, Tohoku Math. J. 15 (1963), 121-139.

[8] Korkmaz B., Curvature and normality of complex contact manifolds, PhD Thesis, Michigan State University East Lansing, MI, USA, 1997.

[9] Korkmaz B., A curvature property of complex contact metric structure, Kyungpook Math. J. 38 (1998), 473-488.

[10] Korkmaz B., Normality of complex contact manifolds, Rocky Mountain J. Math., 30 (2000), 13431380.

[11] Kobayashi, S., Remarks on complex contact manifolds. Proc. Am. Math. Soc. 10 (1959), 164-167.

[12] Klingenberg Jr., W.: Asymptotic curves on real analytic surfaces in C2. Math. Ann. 273(1985), 149162.

[13] LeBrun, C.R., S.M.Salamon, Strong rigidity of positive quaternion-Kähler manifolds. Invent. Math. 118 (1994), 109-132.

[14] Nazimuddin, A.K.M., Ali M. S., Connections with Symplectic Structures. American Journal of Computational Mathematics, 6 (2016), 313-319.

[15] Pöschel, J.: On invariant manifolds of complex analytic mappings near fixed points. Expo. Math. 4 (1986), 97-109.

[16] Stolovitch, L., Family of intersecting totally real manifolds of $\left(\mathbb{C}^{\mathrm{n}}, 0\right)$ and germs of holomorphic diffeomorphisms, Bull. Soc. Math. Fr. 143 (2015), 247-263.

[17] Tischler, D., Closed 2-forms and an embedding theorem for symplectic manifolds, J. Differ. Geom. 12 (1977), 229-235.

[18] Thurston W., Some simple examples of symplectic manifolds, Proc. Amer. Math. Soc. 55 (1976), 467468.

[19] Wolf J. A., Complex homogeneous contact manifolds and quaternionic symmetric spaces, J. Math. and Mech., 14 (1965), 1033-1047. 